\title{
Translating Pharmacomicrobiomics: Three Actionable Challenges/Prospects in 2020
}

\author{
Ramy K. Aziz, ${ }^{1,2}$ Mariam R. Rizkallah, ${ }^{3}$ Rama Saad, ${ }^{4}$ and Marwa T. EIRakaiby ${ }^{1,2}$
}

Introduction

$\mathbf{T}$ HE YEAR 2020 MARKS A DECADE since the term pharmacomicrobiomics was coined (Rizkallah et al., 2010) to crystallize a century-old concept of mutual interactions between humans, drugs, and the microbial world. The human microbiome, with its immense metabolic potential that exceeds and expands the human metabolic capacities, has the ability to modulate pharmacotherapy by affecting both pharmacokinetics and pharmacodynamics of drug molecules:

The simple definition of pharmacomicrobiomics is the (systematic) study of drug-microbiome interactions. More specifically, it is the study of how intra- and inter-individual microbiome variations affect drug action, disposition, efficacy, and toxicity. The emphasis here is on the effect of microbiome (i.e., microbial community) variations on pharmacokinetics and pharmacodynamics of drug therapy, rather than interactions between drugs and individual microbes.

(Aziz, 2018)

Although drug-microbiome interactions have been described for decades, before the Human Microbiome Project (HMP), all such reported interactions were scattered and uncoordinated, as they originated from sporadic studies spread over the years; thus, integrating them was challenging. As the human microbiome research gained momentum and began to define core microbiomes and microbiome variants (enterotypes, vagitypes, and metabotypes), the previously scattered reports seemed to have finally found a research niche that could lead to an exciting systematic field of scholarly inquiry.

What sounded perhaps like science fiction, or a futuristic "glimpse," when the word/concept of pharmacomicrobiomics was coined in 2010 (Rizkallah et al., 2010), is now becoming a concrete reality.

In 2010, we predicted three possible implications of pharmacomicrobiomics for the 2010-2019 decade. Our predictions included the use of personalized probiotics and phage cocktails, both of which are finding applications today to alleviate the adverse effects of chemotherapy or treat recalcitrant infections. We have also proposed resistome scanning or mapping of different human tissues, which may not be an implemented clinical strategy yet, but is being heavily researched and could be coming soon to the clinical practice (Rizkallah et al., 2010).
An imminently pressing idea that has yet to see the light is pharmacomicrobiomic testing. A million dollar question is: Has not the time come for routine microbiome testing and establishing pharmacomicrobiomic guidelines, at least for some drugs, in 2020?

Looking forward to 2020, we highlight three actionable challenges in the field of pharmacomicrobiomics that can transform to prospects for discovery and clinical science innovation.

\section{Actionable Challenge 1}

\section{Systematic high-throughput microbiome screening studies}

With the remarkable advances in sequencing technology, computational biology, and robotics, high-throughput screens became more available to medium-sized research laboratories, allowing academic and start-up research groups to enter the race of systematic screens for lead molecules, antimicrobials, and vaccine candidates.

Another axis that witnessed tremendous advance is that of culturing the microbiota or culturomics (Lagier et al., 2016). Whereas microbiome research started with the notion that the vast majority of microbial life remains uncultured, the past decade witnessed at least a doubling of the number of microbial taxa that can be cultured (Lagier et al., 2016).

The exciting intersection of the aforementioned two technologies (high-throughput sequencing/screening and microbial culturomics) has now made pharmacomicrobiomic and toxicomicrobiomic screening a reality.

An outstanding prototypic study in this premise screened 76 gut microbial taxa against 271 orally administered drugs (Zimmermann et al., 2019), ushering in a new phase of pharmacomicrobiomics that will likely be characterized by routine screens at different phases of drug development (lead discovery and preclinical phases, safety studies, and postmarketing surveys).

\section{Actionable Challenge 2 \\ Phage-enabled precision microbiome engineering/editing}

Although bacteriophages and phage cocktails are already being used at different levels in biocontrol or antimicrobial

\footnotetext{
${ }^{1}$ Department of Microbiology and Immunology, Faculty of Pharmacy, Cairo University, Cairo, Egypt.

${ }^{2}$ Center for Genome and Microbiome Research, Faculty of Pharmacy, Cairo University, Cairo, Egypt.

${ }^{3}$ Department of Biometry and Data Management, Leibniz Institute for Prevention Research and Epidemiology - BIPS, Bremen, Germany.

${ }^{4}$ Department of Pathology, College of Medicine, University of Illinois, Chicago, Illinois.
} 
therapy, precise removal of specific members of the microbiota by bacteriophage is still in its early phases.

In the coming decade, we expect to see bacteriophages used to precisely remove colorectal cancer-causing microbes, to eliminate specific microbes that aggravate adverse effects of chemotherapeutic agents, or as a supportive therapy with some drugs with narrow therapeutic index (e.g., digoxin) to remove bacteria that render it inefficient (e.g., Cgr-encoding Eggerthella lenta; Haiser et al., 2013).

Just a few months ago, an elegant study reported the successful use of a bacteriophage to decrease mortality by alcoholic fatty liver through selective removal of Enterococcus faecalis, which produces cytolysins that lead to deterioration of patients with alcoholic hepatitis (Duan et al., 2019). We see this as a prescient study for the next decade in use of phages for microbiome editing/rewiring.

We predict massive expansion of this microbiome editing field, not only on the community composition level (modulating the microbiota), but also by modulating the microbiome through editing the core and accessory genomes of specific members of the microbiota. This subgenomic-level editing can be transiently achieved through RNA interference (some sort of microbiata gene therapy) or through the use of CRISPR-CAS systems for higher resolution editing of the microbiota (e.g., by removing specific genetic loci, genomic islands, or inserting desired genes).

\section{Actionable Challenge 3}

\section{Pharmacomicrobiomics in the clinic}

We view clinical pharmacomicrobiomics as an imminent overdue measure, especially for some drugs whose pharmacomicrobiomic interactions are well defined [e.g., digoxin (Haiser et al., 2013), irinotecan (Lin et al., 2014), tenofovir (Klatt et al., 2017), and acetaminophen (Clayton et al., 2009)]. Moreover, we foresee an expansion of pharmacomicrobiomic and pharmacogenomic testing, as well as clinical guidelines that will cover major classes of drugs; and we believe that this expansion will mark a pivotal turning point in the history of precision medicine.

\section{Conclusion}

We anticipate that the next decade, which coincides with phase 2 of the HMP (the phase that aims at moving from identification to integration to intervention), will witness both interventional measures (e.g., microbiome editing) and clinical implementation (microbiome testing) that will be guided by a booming number of pharmacomicrobiomic screens, which we also think will soon be a routine step in drug discovery and pharmacovigilance protocols.

Our three suggested challenges will help translate the nascent area of pharmacomicrobiomics from bench to bedside, from bench to clinical laboratories, and from bench to pharmaceutical innovation. Translating pharmacomicrobiomics to routine health care applications is one of the current and actionable million dollar questions within the systems sciences innovation ecosystem.

\section{Author Disclosure Statement}

The authors declare they have no competing financial interests.

\section{Funding Information}

R.K.A. and M.T.E. are supported by the Egyptian Academy for Scientific Research and Technology (ASRT) JESOR program (contract no. 41, 2018). The views expressed are the personal opinions of the authors.

\section{References}

Aziz RK. (2018). Interview with Prof. Ramy K. Aziz, Cairo University. The dawn of pharmacomicrobiomics. OMICS 22, 295-297.

Clayton TA, Baker D, Lindon JC, Everett JR, and Nicholson JK. (2009). Pharmacometabonomic identification of a significant host-microbiome metabolic interaction affecting human drug metabolism. Proc Natl Acad Sci USA 106, 14728-14733.

Duan Y, Llorente C, Lang S, et al. (2019). Bacteriophage targeting of gut bacterium attenuates alcoholic liver disease. Nature 575, 505-511.

Haiser HJ, Gootenberg DB, Chatman K, Sirasani G, Balskus EP, and Turnbaugh PJ. (2013). Predicting and manipulating cardiac drug inactivation by the human gut bacterium Eggerthella lenta. Science 341, 295-298.

Klatt NR, Cheu R, Birse K, et al. (2017). Vaginal bacteria modify HIV tenofovir microbicide efficacy in African women. Science 356, 938-945.

Lagier JC, Khelaifia S, Alou MT, et al. (2016). Culture of previously uncultured members of the human gut microbiota by culturomics. Nat Microbiol 1, 16203.

Lin XB, Farhangfar A, Valcheva R, et al. (2014). The role of intestinal microbiota in development of irinotecan toxicity and in toxicity reduction through dietary fibres in rats. PLoS One 9, e83644.

Rizkallah MR, Saad R, and Aziz RK. (2010). The Human Microbiome Project, personalized medicine and the birth of pharmacomicrobiomics. Curr Pharmacogenomics Person Med 8, 182-193.

Zimmermann M, Zimmermann-Kogadeeva M, Wegmann R, and Goodman AL. (2019). Mapping human microbiome drug metabolism by gut bacteria and their genes. Nature 570, 462-467.

Address correspondence to: Ramy K. Aziz, PhD Department of Microbiology and Immunology Faculty of Pharmacy Cairo University Qasr El-Ainy Street Cairo 11562 Egypt

E-mail: ramy.aziz@gmail.com 